\title{
Photodermatology Contorted
}

Die Sonne ist die wesentliche Energiequelle, welche die Biosphäre der Erde speist, formt und ausrichtet. „Alles ist ihr zu verdanken“ formulierten antike Denker, und sie bleiben unwidersprochen.

Dies betrifft auch ein spätes Produkt der Evolution; nämlich uns, Homo sapiens sapiens, den heutigen Menschen! Dabei sind Haut und Augen die primären Organe, auf welche das Sonnenlicht trifft. Dieses Sonnenlicht aber ist eine polychromatische Strahlung von Lichtquanten, Photonen, unterschiedlicher aber distinkter Quantenenergien und reziprok dazu unterschiedlicher Wellenlängen. Man kann daraus ein solares Spektrum beschreiben, die Globalstrahlung, die auf unsere Oberfläche kommt. Sie trifft auf Biomoleküle, welche an bestimmten Positionen distinkte Energiequanten aufnehmen können, um photochemisch zu reagieren. Man nennt es Photochemie. Bestimmte Energiequanten treffen auf molekulare Positionen, die gerade diese Energie, und keine größere oder kleinere, brauchen, um eine kurzlebige oder stabile photochemische Reaktion zu bewerkstelligen. Dies wird Resonanz genannt. Solche Vorgänge sind sehr sensibel und sie können zudem physikalisch oder biologisch noch verstärkt werden. Sie können nur unscharf und sehr unzureichend miteinander verglichen und gewichtet werden. Man kann also wohl Spektren der Resonanz auf der Energieskala beschreiben, Aktionsspektren werden sie genannt, sie eignen sich aber nur sehr eingeschränkt zum Vergleich. Erst die vielfältigen Einwirkungen verschiedener photochemischen Reaktionen auf die biologischen Substrate, die Zellen, deren Organelle, und deren Verbände zu Geweben, bewirken biologische Effekte, gleichsam als Summe aller photochemischer Abläufe. Sie können schon eher vergleichend gewertet werden. Dies wird Photobiologie genannt, und, auf das Organ unserer Haut fokussiert, Photodermatologie. Erst die biologische Wirkung, sofort und später, der vielfältigen pho- tochemischen Reaktionen, ergeben reale Einsichtsmöglichkeiten, was stabil, was vorübergehend, was essentiell, was randständig ist und ganz besonders, was sich biopositiv und was sich bionegativ auswirkt.

Photodermatologie befasst sich mit Wirkung von Sonnenlicht auf die Haut, und über diese wohl auch auf den gesamten menschlichen Organismus (wieder Photobiologie) und wird zusammen mit allen anderen Umweltseinflüssen, den natürlichen und den von Menschen gemachten, in der „Umweltdermatologie“ zusammengefasst. Nochmals kommen immense Möglichkeiten von Wechselwirkungen ins Spiel, welche die Untersuchungen an Modellen erschweren, die tatsächlichen Ereignisse aber besser abbilden.

Im vergangenen Jahrhundert ist die Messung der Sonnenstrahlung, auch spektral, gelungen und künstliche Lichtquellen vermögen sonnenähnliches, teilspektrales und monochromatisches Licht zu generieren und für Versuche bereitzustellen. Auf allen Ebenen der Photophysik, der Photochemie und der Photobiologie sind durch gezielte und modellhafte Versuche bedeutende, ja bahnbrechende Ergebnisse erreicht worden. Sie können mit den Phänomenen an Zellen, an Geweben, im Tierversuch und an menschlicher Haut vergleichend gewertet werden. Sie finden Bestätigung in den Forschungen bei gendefekten Tieren (z.B. knockout Mäusen) und an erbkranken Menschen (Formen von Albinismus, Xeroderma pigmentosum u. a.), sowie in den Resultaten der epidemiologischen Erhebungen.

Die Bedeutung der Grundpigmentierung für die rassische Segregation zur Bevölkerung der gesamten Erde mitsamt extremen Regionen wird verstanden. Das UV-Erythem (Abb.1) und seine Bedeutung als, zwar imperfekte, Warnvorrichtung zur UV-Karzi- 


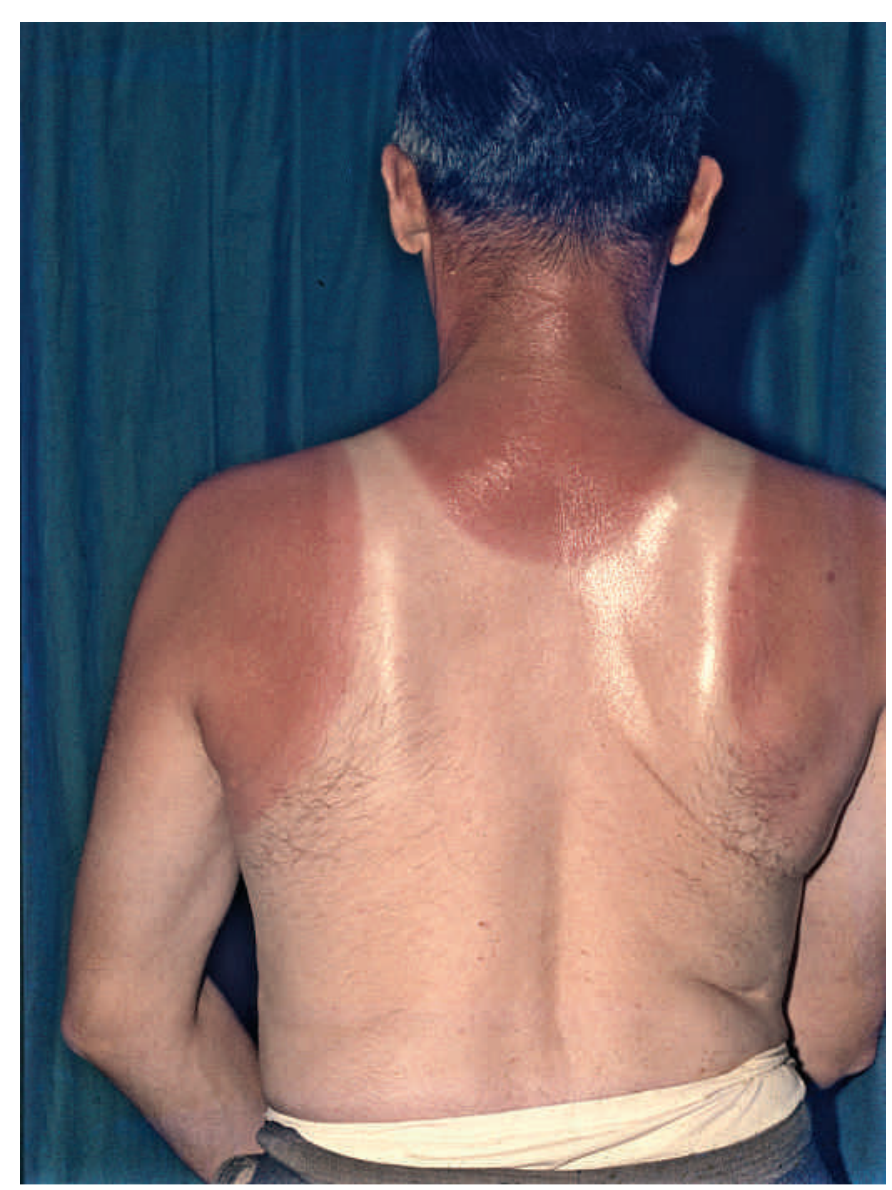

Abb. 1 Unfreiwilliger Sonnenbrand 2 Tage nach der Gartenarbeit an der prallen Mittagssonne. Ca. 8 MED, also die zehnfache Überschreitung der „Schadensdosis“.

nogenese ist verstanden. Die molekularen Reparaturmechanismen und die adaptiven Schutzfunktionen der Haut (Pigmentstimulierung und Lichtschwiele) können eingeschätzt und gewertet werden. Ihre Funktion wie auch ihre Überforderung durch einmalige oder repetitive, überschwellige UV Belastung werden verstanden. Dies gilt auch für die UV induzierte Vitamin D3-Synthese in der Haut und deren Bedeutung für den gesamten Organismus. Man beginnt Photoaging zu verstehen und Photoimmunologie, Photopsychdermatologie und Chronobiologie. Neuerdings werden auch die kanzeroprotektive Wirkung der lichtinduzierten Vitamin D3-Synthese in der Haut, sowie kreislaufaktive und allgemein roborierende Effekte erfolgreich erforscht. Dem steht die große und stetig noch zunehmende Bedrohung durch die UV-Karzinogenese von Melanomen und Plattenepithel-Karzinomen der Haut gegenüber. Wahrlich eine gewaltige Kontroverse!

Fazit ist, dass ein Übermaß an UV-Belastung der Haut das hauptsächlichste Karzinogen für Hauttumoren ist, und dass absolute Karenz von Sonnenlicht menschliches Leben sehr einschränkt und diesem den größten Teil an Lebensqualität entzieht.

Zuviel ist tödlich, sagen die einen mit großer Vehemenz, und vermögen dies mit mannigfachen Befunden zu belegen. Zuwenig ist krankmachend und schränkt Lebensqualität und Entfaltungs- möglichkeit entscheidend ein, meinen die Anderen. Diese vertrackte Situation beherrscht zurzeit die Photodermatologie

Nun zeigt aber die Evolution, insbesondere die Vermehrung und die Verteilung der Menschen in alle Regionen, dass es ein Mittelmass gibt, ja geben muss, welches ein relatives Optimum an biopositiven Effekten zulässt und die Vermeidung der deletären bionegativen, vor allem der karzinogenen Schäden ermöglicht. Es scheint zu genügen, und kaum zu schaden, wenn ein Zehntel der Körperoberfläche für einige Minuten täglich, oder alle 2 Tage, der Sonne ausgesetzt wird. Dabei ist natürlich nicht an das Extrem der „äquatorialen Mittagssonne“ gedacht.

Vehemente Debatten finden statt zwischen den Vertretern der polaren Positionen und deren wissenschaftlich dokumentierten Argumenten. Vergleicht man die Debatte mit solchen der vergangenen Jahrzehnten, die recht sachlich und dem Verbraucher zugewandt abliefen, so muss man neuerdings eine erstaunliche Verschärfung konstatieren. Allzu oft werden die rationalen Positionen verlassen und emotionale Parforcen treten in den Vordergrund. Auch die modernen, scheinbar abgeklärten und naturwissenschaftlichen Grundsätzen verpflichteten Kollegen unterliegen dem was der Zürcher Psychiater Eugen Bleuler schon 1921 das autistisch-undisziplinierte Denken in der Medizin nannte. Während in der frühen Neuzeit abweichende Lehrmeinungen noch einem Gottesurteil unterworfen wurden, sind im 19. Jahrhundert wissenschaftliche Kontroversen in Duellen ausgefochten worden. Und nun das! Zwar entscheidet nicht mehr „die Kugel“, aber die Schärfe des Wortes vermag, gesteigert durch die mediale Kraft, Personen und Werk auch beinahe „tödlich“ zu treffen.

Im Jahre 2002 wurden die Ultraviolette Strahlung (UV-Licht der Sonne und der künstlichen Lichtquellen) in das „National Toxicology Program“ des US-Departement of Health and Human Services aufgenommen. Unglücklich und falsch war, dass die Exposition an sich aufgeführt wurde und nicht die übermäßige Exposition. Denn wir wissen sehr genau und unwidersprochen, dass schon minimale Erythemdosen die hauteigenen Schutz- und Reparaturmechanismen überfordern und, wenn sie repetiert erfolgen, nach Jahren an diesen häufig exponierten Hautbereichen aktinische Keratosen und Plattenepithelkarzinome verursachen. Anderseits wissen wir ebenfalls, dass schon einmalige starke Sonnenbrände, besonders bei der dünnen und empfindlichen Kinderhaut der ersten 15 Lebensjahre, mit dem Auftreten von Melanomen stark korrelieren.

Daran scheiden sich nun die Geister, extreme Positionen werden eingenommen und die „Messer“ gewetzt. Dermatologen, angeführt von der prominenten Forscherin Barbara Gilchrist aus Boston, Toxikologen und andere Wissenschaftler in USA und anderswo vertreten eine extrem bionegative Einschätzung der Sonnenbestrahlung und propagieren eine „Sonnenphobie“. Demgegenüber und im Focus der Anfechtung steht der ebenfalls in Boston tätige und hoch erfolgreiche Endokrinologe Michael Holick, der mit vielen Kollegen die heilsamen, ja schützenden Kräfte des Sonnenlichtes betont und einen moderaten, deutlich unter der Erythemschwelle angesiedelten Sonnengenuss argumentiert und vertritt. 
Gegenseitig werden dieselben Vorwürfe artikuliert: Eitelkeit, Erfolgssucht, Fanatismus, Einseitigkeit, Überwertung eigener Forschung, Uneinsichtigkeit, Scheuklappen gegenüber den Resultaten der Anderen und Vorteilnahme an Renommee sowie finanzieller Unterstützung. Den „Sonnenphobikern“ wird die Verbindung zur Industrie der Sonnenschutzmittel vorgeworfen, den anderen eine solche zu den Herstellern von künstlichen Lichtquellen (Solarien etc.). Im Vordergrund stehen Eitelkeit und Geld.

Es geht also vorwiegend um die Wertung und Konditionierung der Freizeit, der Fernreisen, des Sonnenbadens, des Bräunens und um Schutzmaßnahmen bei Arbeit, Sport, Freizeitgestaltung und „Wellness“. Bemerkenswert ist, und erfreulich, dass die Debatte sich nicht auf die therapeutische Nutzung von UV-Licht bei schweren Krankheiten ausgedehnt hat.

Die Debatte begann und spitzt sich in Boston zu. Freundschaften und wissenschaftliche Kooperationen werden geopfert, institutionelle Kooperationen abgebrochen und gelöst, kooptierte Posten gekündigt und die wissenschaftliche und persönliche Integrität und Glaubwürdigkeit nicht nur angezweifelt, sondern diskriminiert. Die Debatte beschränkt sich keineswegs auf Boston und Fachkreise, nein, sie wird weltweit geführt und über alle verfügbaren Medien. Und die Korporationen mischen sich ein, polarisieren und verschärfen die Gangart. Die „American Academy of Dermatology“ (AAD) nimmt 2004 im Rahmen der Melanomprophylaxe einseitig Stellung für absolute Sonnenkarenz und Sonnenschutzmittel mit hohen Schutzfaktoren. Dem gegenüber erhebt „The Vitamin D Council“ im September 2004 bittere Klage gegen die gefährliche Missdeutung der photochemischen Vitamin D Produktion in der Haut, gegen die Anzweiflung der kanzeroprotektiven Vitamin-D-Effekte und verlangt von Barbara Gilchrist eine Richtigstellung. Angesprochen werden die Verbraucher (oder Meider) des „Zankapfels“ Sonne, also alle Menschen. Sie werden vehement „aufgeklärt“, zur Parteinahme gebeten und sollen gleichsam Schiedsrichter spielen in dem leiden Disput, dem sie zumeist hilflos ausgesetzt sind.
In Europa findet die Debatte erfreulicherweise moderat statt, obschon das 2004 von Michael Holick und dem WissenschaftsJournalisten Mark Jenkins herausgegebene Buch „The UV Advantage“ nun seit April 2005 in der leicht provokanten Übersetzung „Schützendes Sonnenlicht“ vorliegt (Buchbesprechung Seite 393) und wohl reizen möchte. Dazu kommt, dass inzwischen in der internationalen Fachliteratur Publikationen erschienen sind, die Skepsis zu extremen Positionen, welcher Schlagseite auch immer, rechtfertigen (Bericht: Akt. Dermatol. im Juniheft 2005;31: 252-253). Im deutschsprachigen Raum haben die überregionalen Wochen- und Tageszeitungen in erfreulich objektiver, sachlicher und fundierter Weise Berichte zum Thema geboten, welche den Sachstand der Wissenschaft und die daraus zu ziehende Richtlinien zur Anwendung resp. Meidung von Sonne und bestmöglichem Sonnenverhalten enthalten: Spiegel (34/2004), Focus (23/2005), FASZ (20.3.05), NZZ (4.5.05) und die Süddeutsche Zeitung auch am 4.5.05. Dies ist hoch stehender Wissenschaftsjournalismus und besonders bemerkenswert! Hoffentlich gelingt es nun, ausgehend von Europa, die Debatte der Wissenschaft wieder in den Foren und Zeitschriften derselben zu führen, da wo sie unbedingt hin gehört. Zudem ist dringend zu hoffen, dass der Stil, der Ton und die Sprache dieser Debatte wieder akademische Maßstäbe hält und Toleranz, Kenntnisnahme und Verständnis für die Resultate auch der anderen, sowie Vermeidung der Selbstüberschätzung dominieren. Damit kann die dringend nötigte Forschung zügig zur Lösung der offenen Frage vorangetrieben werden, was wiederum korrekte und durchführbare Ratschlägen für die uns anvertrauten Menschen und Patienten zum Ziele hat. Möge sich Vernunft ausbreiten! 